\title{
Mixtures of Lecithin and Bile Salt Can Form Highly Viscous Wormlike Micellar Solutions in Water
}

\author{
Chih-Yang Cheng, ${ }^{\dagger}$ Hyuntaek Oh, ${ }^{\S}$ Ting-Yu Wang, ${ }^{\ddagger}$ Srinivasa R. Raghavan, ${ }^{\S}$ and Shih-Huang Tung* ${ }^{\dagger}$ \\ ${ }^{\dagger}$ Institute of Polymer Science and Engineering and ${ }^{\ddagger}$ Instrumentation Center, National Taiwan University, Taipei 10617, Taiwan \\ ${ }^{\S}$ Department of Chemical and Biomolecular Engineering, University of Maryland, College Park, Maryland 20742, United States
}

\section{Supporting Information}

ABSTRACT: The self-assembly of biological surfactants in water is an important topic for study because of its relevance to physiological processes. Two common types of biosurfactants are lecithin (phosphatidylcholine) and bile salts, which are both present in bile and involved in digestion. Previous studies on lecithin-bile salt mixtures have reported the formation of short, rodlike micelles. Here, we show that lecithin-bile salt

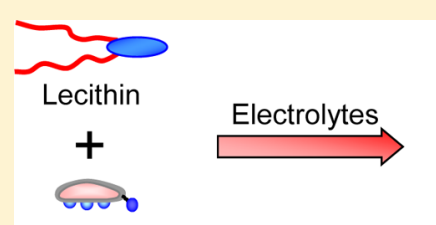

Bile Salts

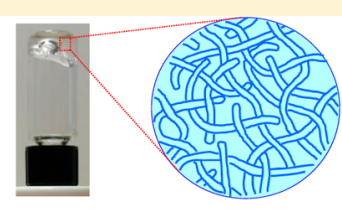

Highly viscous aqueous solutions micelles can be further induced to grow into long, flexible wormlike structures. The formation of long worms and their resultant entanglement into transient networks is reflected in the rheology: the fluids become viscoelastic and exhibit Maxwellian behavior, and their zero-shear viscosity can be up to a 1000-fold higher than that of water. The presence of worms is further confirmed by data from small-angle neutron and X-ray scattering and from cryo-transmission electron microscopy (cryo-TEM). We find that micellar growth peaks at a specific molar ratio (near equimolar) of bile salt:lecithin, which suggests a strong binding interaction between the two species. In addition, micellar growth also requires a sufficient concentration of background electrolyte such as $\mathrm{NaCl}$ or sodium citrate that serves to screen the electrostatic repulsion of the amphiphiles and to "salt out" the amphiphiles. We postulate a mechanism based on changes in the molecular geometry caused by bile salts and electrolytes to explain the micellar growth.

\section{INTRODUCTION}

The self-assembly of biological surfactants in water has been intensely investigated by scientists over the past several decades. Two common classes of such surfactants are phospholipids and bile salts. A typical phospholipid is lecithin, i.e., phosphatidylcholine, which is a zwitterionic molecule with a headgroup having a positively charged choline and a negatively charged phosphate (Figure 1a). In water, lecithin tends to selforganize into bilayer membranes and in turn into vesicles. Bile salts are amphiphiles synthesized in the body within the liver. They have a characteristic steroid structure with a rigid, nonplanar, fused ring and a carboxylic acid group that may be conjugated with amino acids such as taurine and glycine. Different bile salts contain different numbers (one to three) and positions of hydroxyl groups attached to the rings (Figure 1b). Unlike conventional amphiphiles that have a hydrophilic head and hydrophobic tail(s), bile salts are facial amphiphiles with polar and nonpolar faces. ${ }^{1}$ Because of this facial structure, bile salts in water form unusual micelles in which the molecules are packed back-to-back with low aggregation numbers (5$10)$. $^{2}$

The aqueous self-assembly of lecithin/bile salt mixtures is of general interest due to its physiological relevance as well as its biochemical and biomedical applications. ${ }^{1,3-8}$ While lecithin alone forms vesicles, these vesicles are unstable because the bulky hydrophobic tails of the lipid inhibit its solubility in water. Bile salts are much more soluble in water, and in small amounts, they can intercalate into lecithin vesicles and stabilize (a)

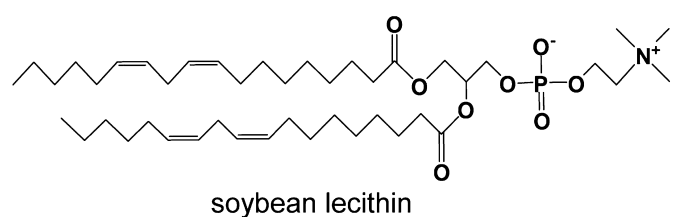

(b)

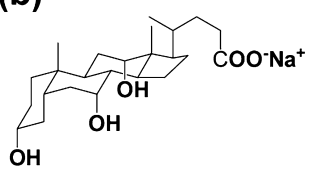

sodium cholate (SC)

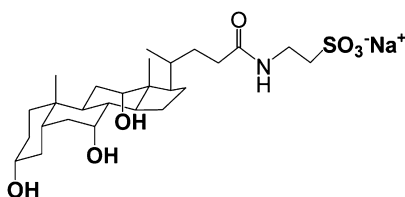

sodium taurocholate (STC)

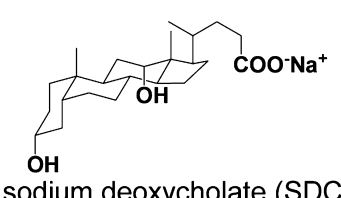

sodium deoxycholate (SDC)

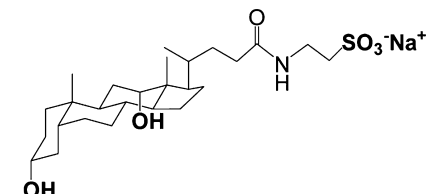

sodium taurodeoxycholate (STDC)
Figure 1. Molecular structures of (a) soybean lecithin and (b) bile salts used in this study.

Received: June 18, 2014

Revised: August 14, 2014

Published: August 14, 2014 
these structures. Accordingly, lecithin-bile salt vesicles have been examined in the context of lipid-protein interactions ${ }^{9}$ and the enzymatic hydrolysis of phospholipids. ${ }^{10}$ At higher amounts, bile salts and lecithin form mixed micelles. Physiologically, mixed micelles of lipids and bile salts are known to play a crucial role in the digestion, intestinal absorption, and transport of dietary fats, including cholesterol. ${ }^{1,3,11}$ From an application standpoint, mixed micelles and vesicles containing bile salts and lecithin have been employed as carriers for delivering drugs across the intestinal walls. ${ }^{12,13}$

The self-assembly of lecithin/bile salt mixtures in water is known to be dependent on the molar ratio of the two species, ${ }^{14,15}$ the total lipid concentration, ${ }^{16}$ and the ionic strength. ${ }^{17}$ These mixtures form micelles at a molar ratio of bile salt to lecithin $\sim 1$. Initial studies suggested that the micelles are disklike in shape. ${ }^{18}$ However, later evidence from light scattering, ${ }^{19}$ small-angle neutron scattering (SANS), ${ }^{15,20}$ cryotransmission electron microscopy (cryo-TEM) ${ }^{14}$ and other ${ }^{21}$ techniques have shown that the micelles are cylindrical in shape. A couple of studies have suggested that the cylinders can sometimes grow into long chains. ${ }^{14,15}$ Cylindrical micellar chains that are long and flexible are termed wormlike micelles ("worms" for short). Much like polymer chains, worms are expected to entangle into a transient network, transforming the solution from a Newtonian, low-viscosity state into a highly viscous and viscoelastic state. ${ }^{22-24}$ The zero-shear viscosity $\eta_{0}$ of semidilute worms $(<10 \mathrm{wt} \%)$ can be several orders of magnitude higher than that of water. ${ }^{25}$ However, to our knowledge, highly viscous worms of lecithin/bile salt in water have not been reported so far. This suggests that the cylindrical lecithin/bile salt micelles prepared in previous studies are relatively short and thereby unable to entangle with one another.

Apart from aqueous systems, mixtures of lecithin and bile salts have also been studied in nonpolar organic solvents (oils), where they show interesting self-assembly. Lecithin alone in oils forms reverse spherical or ellipsoidal micelles. ${ }^{26}$ Bile salts are insoluble in oils, but they can be dissolved in the presence of lecithin. When bile salts are added to lecithin reverse micelles, the micelles grow into long flexible cylinders, which are termed reverse wormlike micelles. The mechanism for this transition is believed to involve binding of the bile salts to the phosphate groups on lecithin, which then alters the effective geometry of the molecules so as to favor long cylinders. ${ }^{26,27}$ Remarkably, the zero-shear viscosity $\eta_{0}$ of these reverse worms can be 5 orders of magnitude higher than that of the parent oil. Thus, it is known that lecithin/bile salt can form long reverse worms in oil; however, "normal" worms of the same in water have not been reported yet.

In this work, we have succeeded in finding a set of conditions that result in highly viscous semidilute solutions of lecithin/bile salt in water. We confirm via rheology, scattering techniques, and cryo-TEM that the high viscosities are due to the formation of wormlike micelles. We show that worms can be obtained using four different bile salts (Figure $1 \mathrm{~b}$ ): the trihydroxy bile salts, sodium cholate (SC) and sodium taurocholate (STC), and the dihydroxy bile salts, sodium deoxycholate (SDC) and sodium taurodeoxycholate (STDC). The formation of worms is maximized at a specific molar ratio of bile salt:lecithin, and in turn the viscosity reaches a peak at this molar ratio. Additionally, the formation of worms also requires a background electrolyte at sufficient levels. This background electrolyte provides an ionic strength to screen the repulsive forces between bile salt anions as well as the Hofmeister effect that "salt out" the bile salts. ${ }^{28}$ We have postulated a mechanism to explain why the above conditions are required for the growth of worms.

Our finding that lecithin/bile salt mixtures can form worms in water is interesting and potentially important. These findings could be relevant for understanding the physiological roles of the different components in bile. It could have implications for diseases of the liver, pancreas, or gall bladder, such as hypercholesterolemia and gallstone formation. ${ }^{29,30}$ Moreover, phospholipid-based gels or viscoelastic fluids have recently been evaluated as candidates for delivering drugs across skin. ${ }^{31,32}$ For instance, lecithin-based reverse worms in oil have been shown to enhance the dermal permeation of drugs. ${ }^{31}$ In a similar vein, lecithin/bile salt based worms in water may also be useful as transdermal carriers of active ingredients that could be released in a controlled manner. In other words, the biocompatibity and viscoelasticity of the system can make it useful in medical or cosmetic formulations.

\section{EXPERIMENTAL SECTION}

2.1. Materials. Soybean lecithin (95\% purity) was purchased from Avanti Polar Lipids, Inc. The bile salts sodium cholate (SC, >99\%), sodium deoxycholate (SDC, $>97 \%$ purity), sodium taurocholate (STC, $>97 \%$ ), and sodium taurodeoxycholate (STDC, >95\%) were purchased from Sigma-Aldrich. Sodium chloride $(\mathrm{NaCl},>99.7 \%)$, calcium chloride dihydrate $\left(\mathrm{CaCl}_{2}\right)$, and sodium citrate dihydrate $(>99.9 \%)$ were purchased from J.T. Baker. Sodium sulfate dihydrate $\left(\mathrm{Na}_{2} \mathrm{SO}_{4},>99.3 \%\right)$ was purchased from Macron Chemicals. Iron chloride $\left(\mathrm{FeCl}_{3},>98 \%\right)$ was purchased from Alfa Aesar. All chemicals were used as received.

2.2. Sample Preparation. Lecithin and bile salts were dissolved in methanol to form $200 \mathrm{mM}$ stock solutions. The molar ratio of bile salt to lecithin was adjusted by mixing appropriate amounts of the stock solutions. After mixing, methanol was removed by drying the samples in a vacuum oven at $50{ }^{\circ} \mathrm{C}$ for $48 \mathrm{~h}$. The final samples with desired amphiphile and electrolyte concentrations were obtained by adding proper amounts of deionized water and electrolytes, followed by stirring and sonication until the solutions were well mixed.

2.3. Rheology. We performed steady and dynamic rheological experiments on an AR2000EX stress controlled rheometer (TA Instruments). Cone-and-plate geometry $\left(40 \mathrm{~mm}\right.$ diameter with $4^{\circ}$ cone angle) was used for all samples. The plate was equipped with Peltier-based temperature control, and all samples were studied at 25 $\pm 0.1{ }^{\circ} \mathrm{C}$. We utilized a solvent trap to minimize the evaporation of water. Dynamic frequency spectra were conducted in the linear viscoelastic regime of the samples, as determined from dynamic strain sweep measurements. For steady-shear experiments, sufficient time was allowed before data collection at each shear rate to ensure that the viscosity reached its steady-state value.

2.4. Cryogenic Transmission Electron Microscopy (CryoTEM). Specimens for cryo-TEM were prepared with a FEI Vitrobot Mark IV vitrification system at $22{ }^{\circ} \mathrm{C}$ and $100 \%$ relatively humidity. Samples were applied onto grids with porous carbon films. After threetime blotting processes, the grids were immediately plunged into liquid ethane and then stored in liquid nitrogen before imaging. TEM imaging were conducted on a FEI Tecnai G2 transmission electron microscope operated at a voltage of $120 \mathrm{kV}$.

2.5. Small-Angle Neutron and X-ray Scattering (SANS and SAXS). SANS measurements were made on the NG-7 $(30 \mathrm{~m})$ beamline at NIST in Gaitherburg, MD. Neutrons with a wavelength of $6 \AA$ were selected. Two sample-detector distances were used to probe a wide range of wave vectors from 0.0035 to $0.5 \AA^{-1}$. Deuterated water $\left(\mathrm{D}_{2} \mathrm{O}\right)$ instead of protonated water was used as solvent to enhance the scattering contrast. SAXS measurements were conducted on the BL23A1 beamline in the National Synchrotron Radiation Research Center (NSRRC), Taiwan. ${ }^{33}$ A monochromatic beam of $\lambda=0.83 \AA$ was used. The scattering patterns were collected on a Pilatus $1 \mathrm{M}-\mathrm{F}$ 
detector over a $q$ range from 0.01 to $0.4 \AA^{-1}$. All the samples were studied at $25{ }^{\circ} \mathrm{C}$. The spectra are shown as plots of the absolute intensity $I$ versus the wave vector $q=4 \pi \sin (\theta / 2) / \lambda$, where $\theta$ is the scattering angle and $\lambda$ is the wavelength.

2.6. SANS and SAXS Modeling. Modeling of SANS and SAXS data was conducted using the analysis package provided by NIST with IGOR Pro software. ${ }^{34}$ For dilute solution of noninteracting scatterers, the scattering intensity $I(q)$ can be modeled in terms of the form factor $P(q)$ of scatterers

$$
I(q)=c P(q)+B
$$

where $c$ is the total concentration of scatterers and $B$ is the background.

For SANS data, we consider form factor model for flexible cylinder with polydispersity radius. The form factor for semiflexible chains of contour length $L$, Kuhn length $b$, and cross-sectional radius $R$ can be represented as the product of the excess scattering length density $\Delta \rho$, cross-section form factor $P_{\mathrm{CS}}$, and wormlike chain form factor $P_{\mathrm{WC}}::^{35,36}$

$$
P(q)=\Delta \rho^{2} P_{\mathrm{CS}}(q, R) P_{\mathrm{WC}}(q, L, b)
$$

The cross-section form factor $P_{\mathrm{CS}}$ can be approximated by the following expression, which is valid for cylindrical chains

$$
P_{\mathrm{CS}}(q, R)=\left[\frac{2 J_{1}(q R)}{q R}\right]^{2}
$$

where $J_{1}(x)$ is the first-order Bessel function given by

$$
J_{1}(x)=\frac{\sin x-x \cos x}{x^{2}}
$$

For cylinders with polydispersity radius, the form factor should be averaged over the radius distribution in the following manner

$$
P_{\mathrm{CS}}\left(q, R_{0}\right)=\int f(R) P_{\mathrm{CS}}(q, R) \mathrm{d} R
$$

where $R_{0}$ is the average cylinder radius, the polydispersity in cylinder radius $f(R)$ is accounted for by a Schulz distribution

$$
f(R)=\left[\frac{z+1}{R_{0}}\right]^{z+1} \frac{L^{z}}{\Gamma(z+1)} \exp \left[-(z+1) \frac{R}{R_{0}}\right]
$$

where $\Gamma$ is the gamma function and $z$ is a parameter related to the width of the distribution. The polydispersity index $p_{\mathrm{d}}$ is given by

$$
p_{\mathrm{d}}=\frac{1}{\sqrt{z+1}}
$$

The form factor $P_{\mathrm{WC}}$ for a wormlike chain with excluded-volume interactions is detailed in the paper by Pedersen and Schurtenberger, ${ }^{36}$ where it was originally derived, and then modified by Chen et al. ${ }^{37}$

Because of the different origin of scattering contrast, the form factor model of core-shell cylinder instead of flexible cylinder was used to fit the SAXS data. The form factor of core-shell cylinders can be represented as ${ }^{38}$

$$
P(q)=\frac{1}{V_{\mathrm{t}}} \int_{0}^{\pi / 2} f^{2}(q, \alpha) \sin \alpha \mathrm{d} \alpha
$$

where

$$
\begin{aligned}
& f(q, \alpha)=2\left(\rho_{\mathrm{c}}-\rho_{\mathrm{s}}\right) V_{J_{0}}\left(\frac{q L_{\mathrm{c}}}{2} \cos \alpha\right) \frac{J_{1}\left(q R_{\mathrm{c}} \sin \alpha\right)}{q R_{\mathrm{c}} \sin \alpha} \\
& \quad+2\left(\rho_{\mathrm{s}}-\rho_{\mathrm{sol}}\right) V_{\mathrm{t}_{0}}\left[q\left(\frac{L_{\mathrm{c}}}{2}+t\right) \cos \alpha\right] \frac{J_{1}\left[q\left(R_{\mathrm{c}}+t\right) \sin \alpha\right]}{\left[q\left(R_{\mathrm{c}}+t\right) \sin \alpha\right]}
\end{aligned}
$$

where $R_{c}$ is the core radius, $L_{c}$ is the core length, $t$ is the shell thickness. $V_{\mathrm{c}}$ and $V_{\mathrm{t}}$ are the core volume and total volume, respectively. $\rho_{\mathcal{c}} \rho_{\mathrm{s}}$, and $\rho_{\text {sol }}$ are scattering length density of core, shell, and solvent, respectively. $\alpha$ is the angle between the cylinder axis and the scattering vector $q . J_{1}(x)$ is the first-order Bessel function and $J_{0}$ is given by

$$
J_{0}(x)=\sin (x) / x
$$

\section{RESULTS AND DISCUSSION}

3.1. Phase Behavior and Rheology. We first discuss the effect of bile salt:lecithin molar ratio, denoted as $B_{0}$, on the rheological properties of lecithin/bile salt mixtures in water at sufficient electrolyte concentration. The zero-shear viscosity $\eta_{0}$ as a function of $B_{0}$ for representative lecithin/SC mixtures at a high $\mathrm{NaCl}$ concentration of $5 \mathrm{M}$ is shown in Figure 2a. The

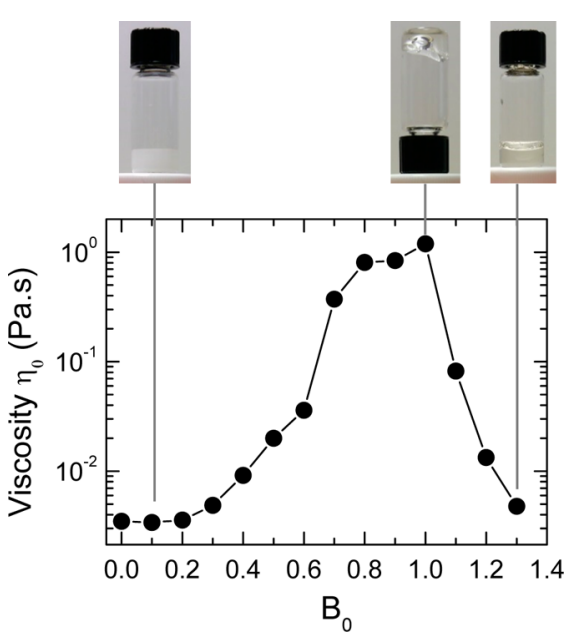

Figure 2. Zero-shear viscosity $\eta_{0}$ as a function of the molar ratio of bile salt to lecithin $B_{0}$ for lecithin/SC mixtures in water. Lecithin concentration is fixed at $100 \mathrm{mM}$, and $\mathrm{NaCl}$ concentration is $5 \mathrm{M}$.

lecithin concentration was fixed at $100 \mathrm{mM}$. At a $B_{0}$ below 0.2 , the viscosity is almost as low as that of water. The low ratio of bile salt is unable to alter the bilayer structure of lecithin so that large aggregates are present in the solution and the solution is turbid and of low viscosity (see photograph of typical sample on the top left). ${ }^{17}$ As $B_{0}$ increases above 0.2 , the viscosity increases rapidly and reaches a maximum at a molar ratio denoted as $B_{0}{ }^{\max }$. The viscosity is about a 1000 times higher than that of water. The sample is transparent and flows slowly in the inverted vial as shown in the photograph, indicating a transformation of bilayers into long cylindrical micelles. Such a high viscosity can only be obtained in a narrow $B_{0}$ range. After $B_{0}{ }^{\max }$, the viscosity drops sharply. At $B_{0} \sim 1.3$, the sample becomes a thin, clear liquid with a viscosity close to that of water, implying that the long micelles have been turned into short ones. The four bile salts shown in Figure 1 exhibit very similar trends in the $\eta_{0}-B_{0}$ relationship, and the $B_{0}{ }^{\max }$ values for SC, SDC, STC, and STDC are 0.9, 1.2, 0.8, and 1.0, respectively. The zero-shear viscosities as a function of $B_{0}$ for SDC, STC, and STDC samples are shown in Figure S1 of the Supporting Information.

Figure 3 shows the zero-shear viscosity $\eta_{0}$ of the four bile salts as a function of $\mathrm{NaCl}$ concentration $[\mathrm{NaCl}]$ in water at 25 ${ }^{\circ} \mathrm{C}$. The lecithin concentration was fixed at $100 \mathrm{mM}$, and the molar ratios of bile salt:lecithin were fixed at the $B_{0}{ }^{\max }$ for each bile salt. At $B_{0}{ }^{\max }$ without the addition of $\mathrm{NaCl}$, the solutions are slightly cloudy and slightly viscous, indicating that bilayers are still present. As $[\mathrm{NaCl}]$ increases, the solutions become transparent and the viscosity is greatly enhanced, indicating a 


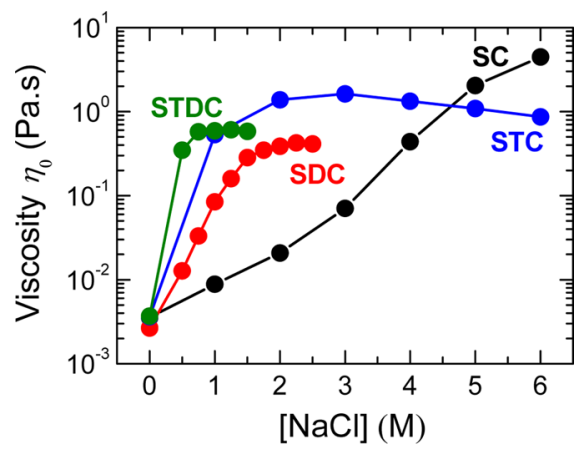

Figure 3. Zero-shear viscosity $\eta_{0}$ of lecithin-bile salt mixtures in water as a function of $\mathrm{NaCl}$ concentration. The lecithin concentration is fixed at $100 \mathrm{mM}$. $B_{0}$ s of SC, SDC, STC, and STDC are 0.9, 1.2, 0.8, and 1.0, respectively. For STDC and SDC, the ends of the curves are the phase boundaries above which the samples phase separate into two liquid phases. For SC and STC, the samples remain homogeneous until the saturated $\mathrm{NaCl}$ concentration $\sim 6 \mathrm{M}$ is reached.

growth of the micelles. For STC, STDC, and SDC samples, the viscosity reaches a plateau, whereas the viscosity of SC samples increases monotonically. Only in the narrow $B_{0}$ range shown in Figure 2 and Figure $S 1$ can we tune the electrolyte concentration to observe this thickening phenomenon. In other words, a specific molar ratio and a sufficient electrolyte concentration are required for the formation of stable, long flexible cylindrical micelles.

With further increase of $\mathrm{NaCl}$ concentration, dihydroxy bile salt STDC and SDC samples phase separate into two liquid phases at $[\mathrm{NaCl}] \sim 1.5$ and $2.5 \mathrm{M}$, respectively, while trihydroxy STC and SC samples remain stable until $[\mathrm{NaCl}]$ is saturated at $\sim 6 \mathrm{M}$. The number of hydroxyl groups on steroid rings of bile salts must play a role in causing the difference. It is known that the aqueous solubility of trihydroxy bile salts is higher than that of dihydroxy ones and that the bile salts conjugated with taurine groups show a lower solubility than unconjugated ones. ${ }^{39}$ The solubility of the four bile salts is therefore in the order of SC > STC > SDC > STDC. The addition of $\mathrm{NaCl}$ screens the repulsive forces between the negatively charged bile salt molecules and thus lowers their solubility in water. ${ }^{40}$ A high $[\mathrm{NaCl}]$ may further decrease the solubility of bile salts due to the interactions of $\mathrm{Na}^{+}$and $\mathrm{Cl}^{-}$ ions with water molecules, known as the Hofmeister effect or the "salting-out" effect. ${ }^{28}$ Since the solubilities of SDC and STDC are lower than those of SC and STC, it is reasonable that the former phase-separate at a lower $[\mathrm{NaCl}]$ than the latter. Also, the $[\mathrm{NaCl}]$ for destabilizing the taurine-based STDC sample is the lowest among the four bile salts.

The viscoelasticity of the lecithin-bile salt mixtures were investigated by dynamic rheology. The elastic modulus $\left(G^{\prime}\right)$ and the viscous modulus $\left(G^{\prime \prime}\right)$ as functions of frequency $\omega$ for trihydroxy SC and STC samples at their maximum viscosities are shown in Figure 4. In each case, the $G^{\prime}$ and $G^{\prime \prime}$ curves intersect, and we see an elastic response at high frequencies and a viscous one at low frequencies. This type of response is characteristic of viscoelastic samples. We fit the data using a Maxwell model with a single relaxation time, which is shown as solid lines through the data:

$$
G^{\prime}(\omega)=\frac{G_{\mathrm{p}} \omega^{2} t_{\mathrm{R}}{ }^{2}}{1+\omega^{2}{t_{\mathrm{R}}}^{2}}
$$
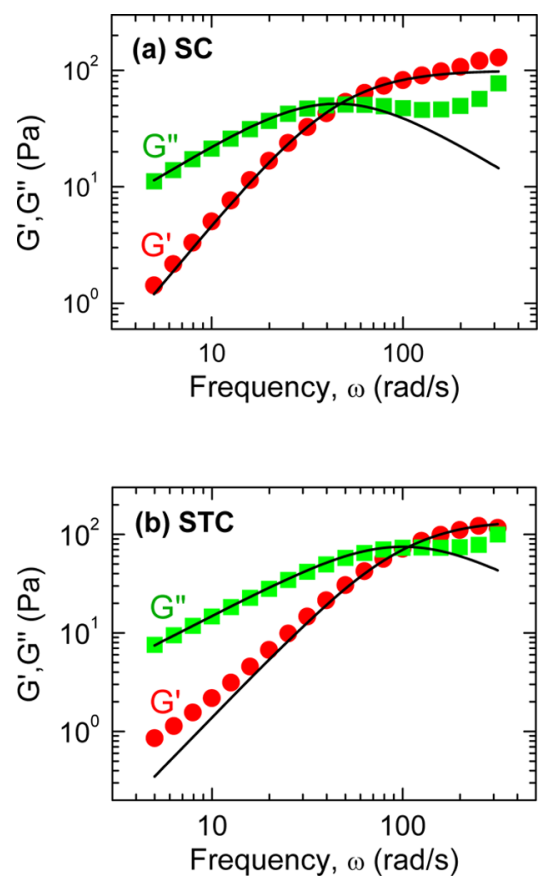

Figure 4. Dynamic rheology data of (a) lecithin/SC mixture at $B_{0}=$ 0.9 and $[\mathrm{NaCl}]=6 \mathrm{M}$ and $(\mathrm{b})$ lecithin $/ \mathrm{STC}$ mixture at $B_{0}=0.8$ and $[\mathrm{NaCl}]=2 \mathrm{M}$ in water at $25^{\circ} \mathrm{C}$. The lecithin concentrations of both samples are $100 \mathrm{mM}$. The plots show the elastic modulus $G^{\prime}$ and viscous modulus $G^{\prime \prime}$ as functions of frequency $\omega$. Fits to singlerelaxation time Maxwell model are shown as black solid lines.

$$
G^{\prime \prime}(\omega)=\frac{G_{\mathrm{p}} \omega t_{\mathrm{R}}}{1+\omega^{2}{t_{\mathrm{R}}}^{2}}
$$

Here $G_{\mathrm{p}}$ is the plateau modulus and $t_{\mathrm{R}}$ is the relaxation time. The data can be fitted reasonably well, especially for the SC sample. In the cases of dihydroxy SDC and STDC (Figure S2 in the Supporting Information), $G^{\prime \prime}$ is larger than $G^{\prime}$ over the whole frequency range. This implies that the micellar chains are less restricted by entanglements, and the samples therefore remain liquid-like.

In addition to $\mathrm{NaCl}$, we have also investigated the effects of different types of electrolytes. The zero-shear viscosity data of the four lecithin/bile salt mixtures at their $B_{0}{ }^{\max } \mathrm{s}$ in the presence of sodium sulfate $\left(\mathrm{Na}_{2} \mathrm{SO}_{4}\right)$ and sodium citrate $\left(\mathrm{Na}_{3} \mathrm{C}_{6} \mathrm{H}_{5} \mathrm{O}_{7}\right)$ are shown in Figure 5a,b. Similar to $\mathrm{NaCl}$, both these electrolytes can effectively increase the viscosity. The efficiency of the three sodium salts in increasing viscosity is in the order $\mathrm{Na}_{3} \mathrm{C}_{6} \mathrm{H}_{5} \mathrm{O}_{7}>\mathrm{Na}_{2} \mathrm{SO}_{4}>\mathrm{NaCl}$. This order is consistent with the greater ionic strengths of sodium citrate and sodium sulfate that more efficiently screen the repulsion between bile salt anions at the same electrolyte concentrations. ${ }^{41}$ Another effect is that citrate and sulfate are known for their ability to "salt out" solutes from water; i.e., they occur on one end of the Hofmeister series. ${ }^{42}$ That is, citrate and sulfate are strongly hydrated anions that reinforce the interactions between water molecules and in turn mitigate the interactions between water and various solutes. Note that $\mathrm{NaCl}$ also shows a salting-out ability at much higher concentrations. Thus, the effect of background electrolyte in our system is consistent with the ability of specific ions to salt out solutes from water. Also, we should point out that the ends of the curves in Figure 5 are the phase boundaries beyond which the samples become turbid. The electrolyte concentration at this 

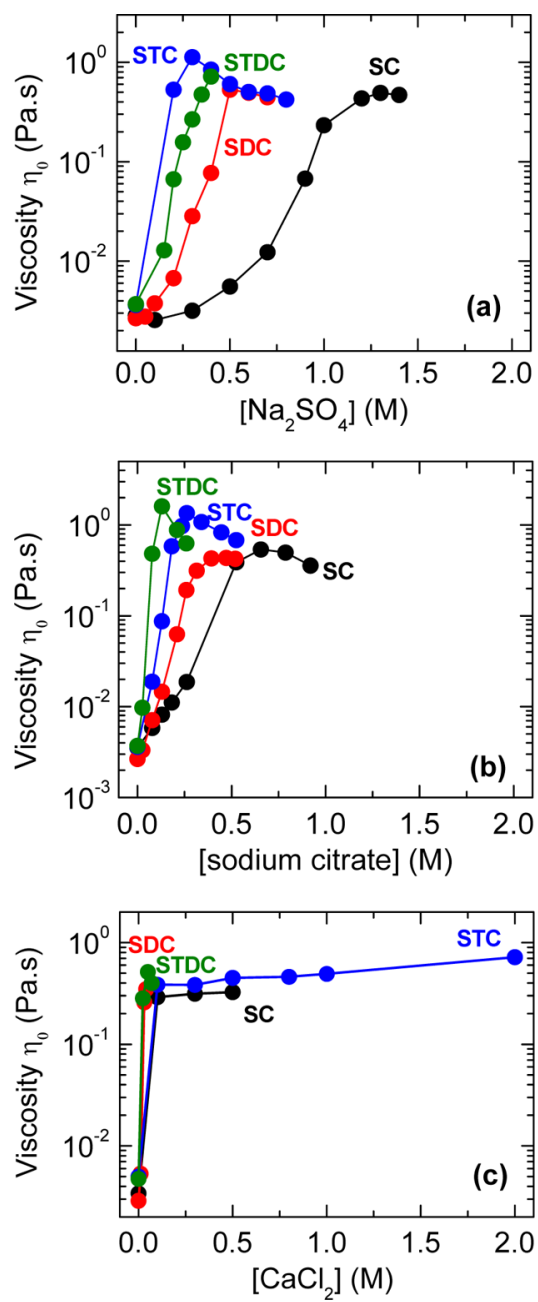

Figure 5. Zero-shear viscosity $\eta_{0}$ of the four lecithin/bile salt mixtures in the presence of varying electrolytes: (a) sodium sulfate $\left(\mathrm{Na}_{2} \mathrm{SO}_{4}\right)$, (b) sodium citrate $\left(\mathrm{Na}_{3} \mathrm{C}_{6} \mathrm{H}_{5} \mathrm{O}_{7}\right)$, and (c) calcium chloride $\left(\mathrm{CaCl}_{2}\right)$. $B_{0}$ s are $0.9,1.2,0.8$, and 1.0 for SC, SDC, STC, and STDC samples, respectively.

boundary follows the order $\mathrm{NaCl}>\mathrm{Na}_{2} \mathrm{SO}_{4}>\mathrm{Na}_{3} \mathrm{C}_{6} \mathrm{H}_{5} \mathrm{O}_{7}$, which is again consistent with the ionic strength and hydrated anions reducing solubility, leading to phase separation at a lower electrolyte concentration.

We also studied electrolytes with multivalent cations, i.e., calcium chloride $\left(\mathrm{CaCl}_{2}\right)$ and iron chloride $\left(\mathrm{FeCl}_{3}\right)$. From the viscosity data shown in Figure 5c, the efficiency of divalent $\mathrm{Ca}^{2+}$ in increasing viscosity is much higher than that of monovalent $\mathrm{Na}^{+}$; i.e., the viscosity maximum is reached at a much lower $\mathrm{CaCl}_{2}$ concentration, $\sim 0.1 \mathrm{M}$, for all four bile salts. The phase separation boundaries also appear at low $\mathrm{CaCl}_{2}$ concentrations, in agreement with previous reports that calcium bile salts are in general less soluble in water than their sodium counterparts. ${ }^{43}$ When iron chloride $\left(\mathrm{FeCl}_{3}\right)$ is added to the mixtures, a trace of trivalent $\mathrm{Fe}^{3+}$ immediately turn the solutions into turbid, and no significant increase in viscosity was observed (data not shown). These results reflect a combination of two aspects. First, cations of higher valency are more effective at screening electrostatic repulsions of bile salt anions in solution. ${ }^{44}$ Another possibility is that some multivalent cations, such as $\mathrm{Ca}^{2+}$, may interact strongly with the negatively charged phosphate groups of lecithin and cause aggregation of lecithin molecules. ${ }^{43,46}$
3.2. SANS, SAXS, and Cryo-TEM Data. We utilized SANS, SAXS, and cryo-TEM to elucidate the self-assembled structures of lecithin-bile salt mixtures in aqueous solutions. The lecithin concentration of the samples was fixed at $20 \mathrm{mM}$, which is a relatively low value, so as to reduce the micellar volume fraction and thereby reduce intermicellar interactions. Representative SAXS data for an STDC sample at $B_{0}=1.0$ without the addition of electrolytes are shown in Figure S3a of the Supporting Information. The sample is slightly cloudy, and the $q^{-2}$ decay of intensity in the intermediate $q$ range indicates the presence of bilayer structures, ${ }^{35}$ which is confirmed by the cryo-TEM image shown in Figure S3b where stacks of bilayers can be clearly seen.

To probe the structures in the viscous regime, we obtained SANS spectra on four samples corresponding to SC, SDC, STC, and STDC, with their respective $B_{0}=0.9,1.2,0.8$, and 1.0 and $[\mathrm{NaCl}]=5.5,0.5,1.0$, and $0.5 \mathrm{M}$. Note that SANS measurements were conducted at a low concentration of amphiphiles where the relative amount of bile salts available for solubilizing the lecithin may decrease because the solutions tend to maintain the concentration of free bile salts in water. ${ }^{47}$ The choice of lower $[\mathrm{NaCl}] \mathrm{s}$ for SANS measurements is to ensure the dilute solutions are stable. $\mathrm{D}_{2} \mathrm{O}$ was used as the solvent for these samples. Neutron scattering originates from the contrast in neutron density between the micelles (protonated molecules) and the solvent (deuterated water), and some water molecules may penetrate into the hydrophilic parts of the micelles. The SANS data are shown in Figure 6a as plots of scattered intensity $I$ vs wave vector $q$. All four samples exhibit a scaling of $I \sim q^{-1}$ at intermediate $q$, which is indicative of cylindrical micelles. ${ }^{35,48}$ We fitted the data using the flexible cylinder model with polydisperse radius (eqs 2-7), and the fits are shown as solid lines through the data. The data are fitted well by the model, indicating the presence of wormlike micelles in the solutions. The fitting parameters from the modeling are listed in Table 1 . The radii of the mixed wormlike micelles are nearly the same, $\sim 20 \AA$, independent of the type of bile salts. Because of the limit of $q$ range in this study and the low amphiphile concentrations used in SANS measurements, the contour lengths listed in Table 1 may not be the exact sizes of the micelles and may not coincide with the trend of viscosity measured at high amphiphile concentrations. The representative cryo-TEM micrograph of a STDC sample prepared in the same manner is shown in Figure 7. The image reveals the presence of long and flexible cylindrical micelles, in agreement with the results from SANS.

Figure $6 \mathrm{~b}$ shows the SAXS data on the same samples, and these are very different from the SANS data. We can see clear humps at $q$ around $0.15 \AA^{-1}$ that are absent in SANS, especially for SDC, STC, and STDC. The contrast in X-ray scattering results from the interactions of incident $\mathrm{X}$-rays with the electrons in the various species. In the present case, the micellar solutions contain three domains with distinguishable electron densities: the hydrophobic core of the micelles, the hydrophilic shell of the micelles, and the solvent $\left(\mathrm{H}_{2} \mathrm{O}\right)$. Therefore, the SAXS data are expected to reflect a core-shell structure for the micelles, unlike the uniform structure extracted from SANS data. We have fitted the SAXS data with the model for coreshell cylinders (eqs 8-10). Fits to the model are shown as solid lines, and these fit the data reasonably well. The fitting parameters are listed in Table 1. For SDC, STC, and STDC samples, the core radius is $8-10 \AA$ and the shell thickness is 20-21 $\AA$. The core is composed of flexible lecithin tails while 

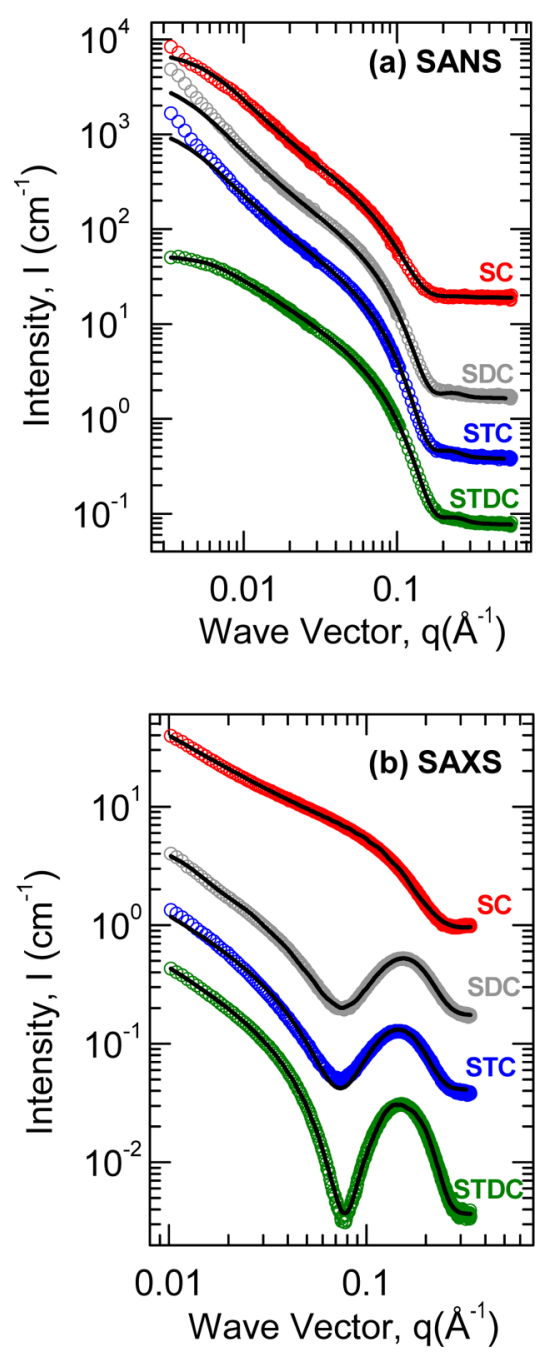

Figure 6. (a) SANS and (b) SAXS data for lecithin/bile salt mixtures at $25{ }^{\circ} \mathrm{C}$. The lecithin concentration is fixed at $20 \mathrm{mM}$ for all the samples. $B_{0}$ values are $0.9,1.2,0.8$, and 1.0 , and $\mathrm{NaCl}$ concentrations are 5.5, 0.5, 1.0, and 0.5 M for SC, SDC, STC, and STDC samples, respectively. Model fits are shown as solid lines through each data.

the shell is composed of lecithin headgroups, bile salts, and maybe some water with electrolyte ions. Both the core and shell sizes are larger for the SC sample, and it should be also noted that for the SC sample the hump at $q \sim 0.15 \AA^{-1}$ is nearly absent, indicating a loss of core-shell characteristics. The larger cross-sectional size of SC mixed micelles and the absence of the hump from the SAXS data are likely due to the high $\mathrm{NaCl}$ concentration $(5.5 \mathrm{M})$ in this sample. Such a high $\mathrm{NaCl}$ concentration may alter the electron density in the hydrophilic shell of the micelles as well as in the solvent.

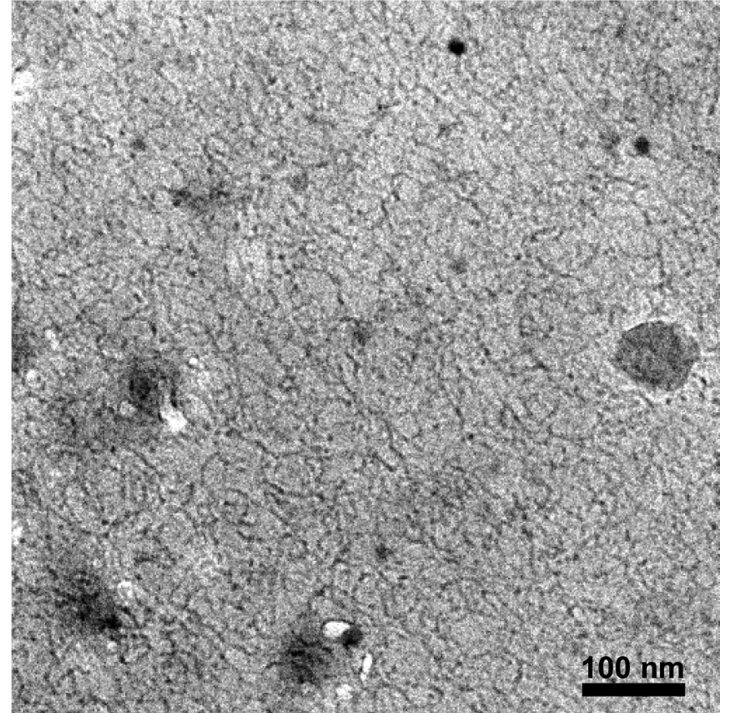

Figure 7. Cryo-TEM image of lecithin/STDC sample at $B_{0}=1.0$ and $[\mathrm{NaCl}]=0.5 \mathrm{M}$. The sample is found to contain long wormlike micelles.

We also utilized the indirect Fourier transformation (IFT) technique to analyze the SAXS and SANS data. IFT gives the pair-distance distribution function $p(r)$ corresponding to each $I(q)$ data, and these are shown in Figure 8 . The $p(r)$ corresponding to the SANS data for all four mixed micelles show an asymmetrical shape (Figure 8a), with an inflection point followed by a decrease to zero. This $p(r)$ function is characteristic of cylindrical micelles. ${ }^{49}$ The inflection point gives the approximate cylinder diameter, while the point where $p(r)$ meets the $r$-axis is an estimate for the average length of the cylinders. Note that if the cylinders are rigid, $p(r)$ should show a linear decrease. The shoulders at $r \sim 100 \AA$ are possibly due to the flexible nature of the micelles. Figure $8 \mathrm{~b}$ shows the $p(r)$ functions corresponding to the SAXS data. At low $r$, the $p(r)$ of SDC, STDC, and STC samples exhibit pronounced maxima and minima, which are regarded as features of core-shell structures. $^{50-52}$ Similar to $p(r)$ from SANS, asymmetric decreases to zero can be seen for all the four bile salts. The inflection point between the first maximum and minimum reflects the cross-sectional sizes of the hydrophobic cores while the inflection point after the second maximum gives the cylinder diameter. The lengths of the cylinders can similarly be estimated from the largest $r$ values where $p(r)$ approaches zero. Note that for the SC sample, due to its indistinctive core-shell feature, the second maximum almost disappears, and the $p(r)$ profile is similar to that of a regular cylinder.

It has been shown that at a fixed $\mathrm{NaCl}$ concentration the viscosity reaches a maximum and then decreases with

Table 1. Fitting Parameters from SANS and SAXS Modeling

\begin{tabular}{|c|c|c|c|c|c|c|c|}
\hline \multirow[b]{3}{*}{ sample } & \multicolumn{4}{|c|}{ SANS } & \multicolumn{3}{|c|}{ SAXS } \\
\hline & av radius & Kuhn length & contour length & polydispersity & core radius & shell thickness & core length \\
\hline & $R_{0}(\AA)$ & $b(\AA)$ & $L(\AA)$ & $p_{\mathrm{d}}$ & $R_{c}(\AA)$ & $t(\AA)$ & $L_{\mathrm{c}}(\AA)$ \\
\hline SC & 19.6 & $196.4 \pm 2.4$ & $1678.1 \pm 18.1$ & 0.14 & 12.7 & 22.4 & $4647.1 \pm 2.8$ \\
\hline SDC & 20.4 & $226.3 \pm 1.5$ & $2865.7 \pm 212.5$ & 0.17 & 8.1 & 21.4 & $368.5 \pm 1.1$ \\
\hline STC & 21.1 & $311.6 \pm 13.1$ & $2607.5 \pm 251.5$ & 0.17 & 9.4 & 21.5 & $1599.9 \pm 5.1$ \\
\hline STDC & 19.4 & $680.6 \pm 57.5$ & $579.3 \pm 4.3$ & 0.16 & 9.9 & 19.9 & $7752.2 \pm 5.4$ \\
\hline
\end{tabular}




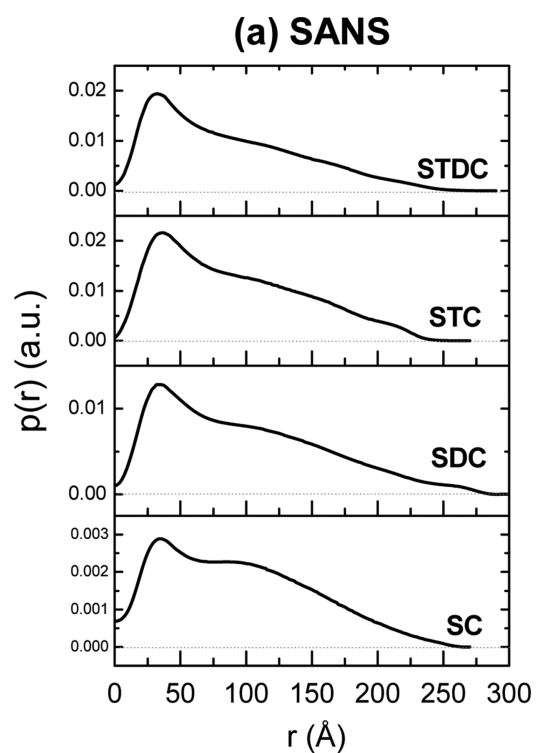

(b) SAXS

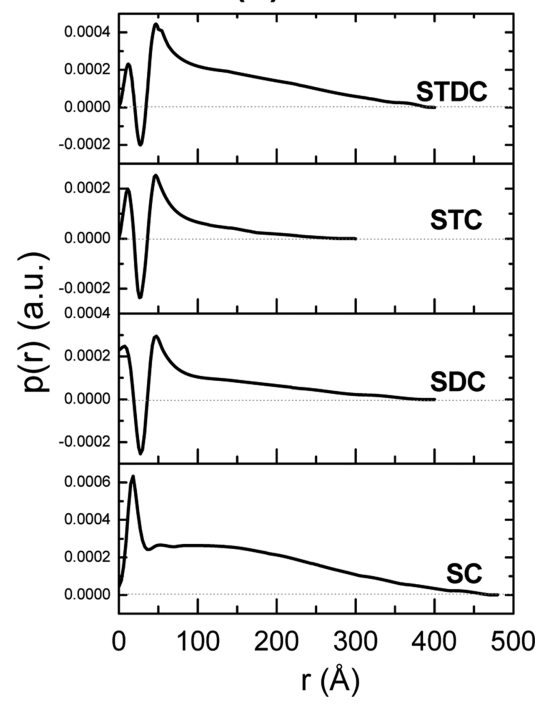

Figure 8. Pair-distance distribution functions $p(r)$ obtained by IFT analysis of the SANS and SAXS data shown in Figure 6.

increasing $B_{0}$, and the samples remain homogeneous and transparent, as shown in Figure 2. The SANS data of STC samples with $B_{0}=0.8,1.0$, and 1.1 at $[\mathrm{NaCl}]=1.0 \mathrm{M}$ are shown in Figure 9. Among these samples, $B_{0}=0.8$ shows the maximum viscosity $\left(\eta_{0}=1 \mathrm{~Pa} \cdot \mathrm{s}\right)$ whereas the viscosities at $B_{0}=$ 1.0 and 1.1 are much lower $\left(\eta_{0}<0.01 \mathrm{~Pa} \cdot \mathrm{s}\right)$. Figure 9 shows that the scattering intensity at low $q$ is dramatically lower for the $B_{0}=1.0$ and 1.1 samples relative to the $B_{0}=0.8$ sample. The decrease in low- $q$ scattering implies a shortening of the micelles. ${ }^{53}$ The SANS data thus confirm that a transition from long to short cylindrical micelles accounts for the viscosity decrease beyond the peak in lecithin/bile salt mixtures. Figure S4 shows the pair-distance distribution functions corresponding to Figure 9 where the shortening in length of wormlike micelles with increasing $B_{0}$ can be clearly seen. Note that the intensity at high $q$ remains practically unchanged, indicating that the crosssectional size of the micelles is nearly the same for different $B_{0}$.

3.3. Mechanism. In the preceding sections, we have shown that with sufficient electrolyte concentration the addition of bile salt can induce a growth of lecithin micelles in water. The

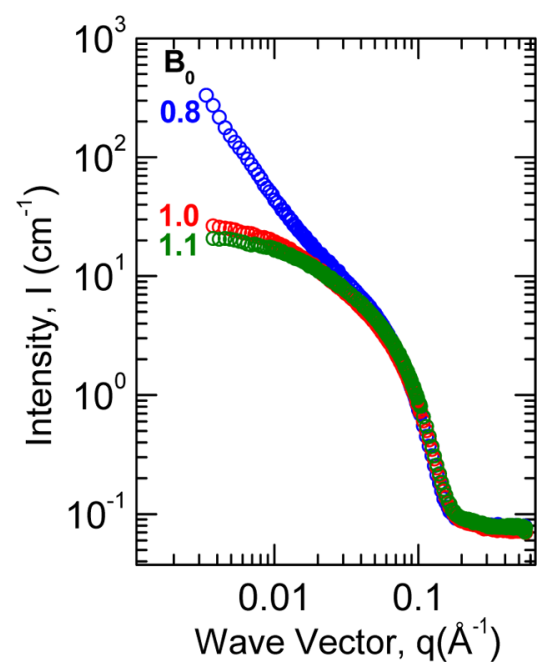

Figure 9. SANS data of lecithin/STC samples at $B_{0} \geq B_{0}{ }^{\max }(\sim 0.8)$. The lecithin concentration is $20 \mathrm{mM}$, and the $\mathrm{NaCl}$ concentration is $1.0 \mathrm{M}$. The intensity at low $q$ greatly decreases with increasing $B_{0}$, indicating long cylindrical micelles are transformed into short ones.

presence of long wormlike micelles significantly enhances the viscosity and imparts viscoelastic character to the samples. In the current section, we will discuss why these conditions are crucial for the micellar growth.

As described in the Introduction, in aqueous solution, the addition of bile salt transforms lecithin vesicles or bilayers into cylindrical mixed micelles. The shape of self-assembled structures is governed by the effective geometry of the amphiphiles, which can be simply expressed by the critical packing parameter $p=v_{\text {tail }} /\left(a_{\mathrm{hg}} l_{\text {tail }}\right)$, where $v_{\text {tail }}$ is the volume of tail, $a_{\mathrm{hg}}$ is the head group area, and $l_{\text {tail }}$ is the tail length. ${ }^{54}$ The transition from bilayers to cylindrical micelles implies a reduction in packing parameter from ca. 1 to $1 / 2$. In the lecithin/bile salt case, this is achieved by an increase in effective headgroup area due to the insertion of bile salts adjacent to the lecithin headgroups within the self-assembled aggregates, as illustrated in Figure 10. It is most likely that the carboxylate (SC and SDC) or sulfonate (STC and STDC) groups of bile salts expose to water and provide the major contribution to stabilizing the aggregates. We expect bile salts to utilize the hydroxyl groups on their steroid rings to form hydrogen bonds with the phosphates on lecithin. Since bile salts are facial amphiphiles, a back-to-back arrangement of bile salts is favorable because this form can prevent the hydrophobic faces of bile salts from contacting water. Such a model is consistent with that proposed by Madenci et al., ${ }^{15}$ and it is also consistent with the core-shell structure deduced from SAXS analysis.

Although lecithin/bile salt cylindrical micelles can be formed, the length of such micelles (without sufficient background electrolytes) is limited and unable to thicken the solutions. Why is the length limited? This can probably be attributed to the distribution of lecithin and bile salts in the micelles. It has been suggested that the distribution of lecithin and bile salt molecules in mixed micelles is not uniform. ${ }^{15}$ The two-tail lecithin molecules with a nearly cylindrical molecular shape prefer to locate in the low-curvature cylindrical bodies while bile salts that generally form highly curved small micelles in water are expected to form the hemispherical end-caps of the cylinders, as shown by the schematic in Figure 10. In other 


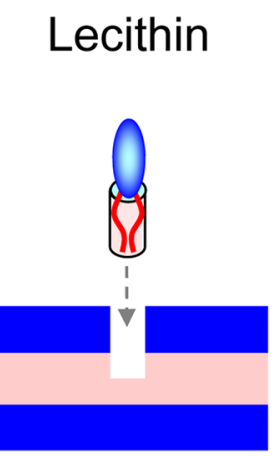

bilayer
Lecithin + Bile Salt

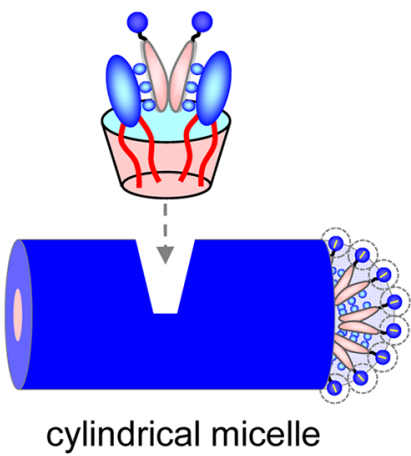

Figure 10. Schematic of the self-assembled structures formed by lecithin with and without bile salt in water. Lecithin is shown as a molecule with a blue head and two red tails, while the bile salt is represented as a facial surfactant. Lecithin alone in water has a critical packing parameter $\sim 1$ and tends to form bilayers. When bile salts are incorporated, they bind to the lecithin headgroups in a back-to-back form, thus expanding the head group area. This alters the net geometry from a cylinder to a truncated cone and thereby induces the bilayers to turn into cylinders. At a low ionic strength, the negatively charged carboxylates or sulfonates of bile salts are highly repulsive, and thus bile salts prefer to pack in the curved hemispherical end-caps of the cylinders.

words, the end-caps of the cylindrical micelles are stabilized by bile salts. When the end-caps are stable, the cylindrical micelles lack a driving force for continued growth into long chains. With more bile salts added into solutions, more end-caps are formed and the cylinders are getting shorter. This explains why a high $B_{0}$ (beyond the peak in Figure 2) leads to the formation of shorter cylindrical micelles, as confirmed by the SANS experiments in Figure 9.

To obtain long wormlike micelles, $B_{0}$ should be sufficiently high to transform lecithin bilayers into cylindrical mixed micelles (i.e., around 1), but it cannot be much higher because it would lead to the formation of redundant end-caps. In the present study, at $B_{0}{ }^{\max }$ of each bile salt without the addition of electrolytes, the amount of the bile salts is not enough to transform the bilayers into micelles so that the samples are still cloudy and of low viscosity, as shown in Figure 3 and Figure S3, but this ratio is very close to the transition boundary. In a micellar solution, the surfactants in micelles are in equilibrium with the free surfactants in the bulk. It is known that the critical micelle concentration (CMC) of ionic surfactants decreases with increasing ionic strength because the repulsive forces between surfactant headgroups are screened. ${ }^{44}$ The Hofmeister salting-out effect of the added electrolytes also causes the CMC of surfactants to decrease because the ions strengthen the hydrophobic effect that drives the micellization in water. ${ }^{55}$ In other words, when electrolyte concentration is increased, more originally free surfactants self-organize into micelles. ${ }^{40,43}$ Following this scenario, the addition of electrolytes into lecithin/bile salt solutions at $B_{0}{ }^{\max }$ should force more originally free bile salt molecules into micelles, which facilitates the transformation of the bilayers into cylindrical micelles and turns the solutions to be transparent.

The effect of electrolytes at $B_{0}{ }^{\max }$ not only induces the formation of cylindrical micelles from bilayers but also promotes the growth of cylindrical micelles into long wormlike micelles. To increase the length of the cylindrical micelles, the end-caps must be destabilized. We suggest that the added electrolytes play a role in this regard. It has been shown that the aggregation number of bile salt micelles in water increases with increasing ionic strength due to the weakening of the electrostatic repulsion between bile salts, ${ }^{56}$ which implies that the curvature of the bile salt micelles is reduced at a high ionic strength. Thus, with an increase in electrolyte concentration, bile salt molecules no longer tend to favor the highly curved end-caps of the cylindrical mixed micelles. This allows the continuous growth of cylindrical micelles into chains that are long enough to increase viscosity. As $B_{0}$ exceeds $B_{0}{ }^{\max }$, the excess bile salts may inevitably form stable end-caps that limit the length of the micelles even at a high electrolyte concentration. Also, more bile salts may further expand the headgroup and reduce the effective packing parameter to a value below $1 / 2$ that favors more curved structures like spherical micelles. This is why only in a narrow $B_{0}$ range can we tune the concentration of electrolytes to obtain highly viscous or viscoelastic solutions of lecithin/bile salt mixed micelles.

\section{CONCLUSIONS}

In this study, we have shown that mixtures of the physiological lipids, lecithin and bile salt, can self-assemble into flexible wormlike micelles in water. Micellar growth has been demonstrated with four different bile salts. The wormlike micelles are sufficiently long to entangle with one another and form highly viscous and viscoelastic fluids. Such elongated aggregates particularly occur under the following conditions: (a) when the molar ratio of bile salt:lecithin is near equimolar and (b) when a sufficient concentration of background electrolyte is used. The transformation of the original lecithin bilayers to cylindrical micelles upon the addition of bile salts is attributed to a change in molecular geometry caused by the binding of bile salt molecules to lecithin headgroups. The study of these mixed micelles could provide insights into physiological processes involving lecithin, bile salt, and electrolytes. In addition, these low-cost biocompatible viscoelastic fluids may have potential for biomedical and cosmetic applications.

\section{ASSOCIATED CONTENT}

\section{Supporting Information}

Zero-shear viscosities as a function of $B_{0}$ for SDC, STC, and STDC samples, dynamic rheology of SDC and STDC samples, SAXS data and cryo-TEM image of STDC sample without the addition of electrolytes, and pair-distance distribution function corresponding to Figure 9. This material is available free of charge via the Internet at http://pubs.acs.org.

\section{AUTHOR INFORMATION}

\section{Corresponding Author}

*E-mail: shtung@ntu.edu.tw (S.-H.T.).

\section{Notes}

The authors declare no competing financial interest.

\section{ACKNOWLEDGMENTS}

This work was financially supported by the grant of the Ministry of Science and Technology, Taiwan (NSC 103-2923E-002-005-MY3). We acknowledge NSRRC, Taiwan, for facilitating the SAXS experiments and NIST, USA, for SANS experiments. 


\section{REFERENCES}

(1) Hofmann, A. F.; Small, D. M. Detergent properties of bile salts correlation with physiological function. Annu. Rev. Med. 1967, 18, 333-376.

(2) Coello, A.; Meijide, F.; Núñez, E. R.; Tato, J. V. Aggregation behavior of bile salts in aqueous solution. J. Pharm. Sci. 1996, 85, 915.

(3) Carey, M. C.; Small, D. M. Characteristics of mixed micellar solutions with particular reference to bile. Am. J. Med. 1970, 49, 590608.

(4) Fahey, D. A.; Carey, M. C.; Donovan, J. M. Bile acid/ phosphatidylcholine interactions in mixed monomolecular layers differences in condensation effects but not interfacial orientation between hydrophobic and hydrophilic bile-acid species. Biochemistry 1995, 34, 10886-10897.

(5) Heuman, D. M. Distribution of mixtures of bile salt taurine conjugates between lecithin-cholesterol vesicles and aqueous media: an empirical model. J. Lipid Res. 1997, 38, 1217-1228.

(6) Ollila, F.; Slotte, J. P. A thermodynamic study of bile salt interactions with phosphatidylcholine and sphingomyelin unilamellar vesicles. Langmuir 2001, 17, 2835-2840.

(7) Elsayed, M. M. A.; Cevc, G. The vesicle-to-micelle transformation of phospholipid-cholate mixed aggregates: a state of the art analysis including membrane curvature effects. Biochim. Biophys. Acta, Biomembr. 2011, 1808, 140-153.

(8) Kupetz, E.; Preu, L.; Kunick, C.; Bunjes, H. Parenteral formulation of an antileishmanial drug candidate - Tackling poor solubility, chemical instability, and polymorphism. Eur. J. Pharm. Biopharm. 2013, 85, 511-520.

(9) Almog, S.; Kushnir, T.; Nir, S.; Lichtenberg, D. Kinetic and structural aspects of reconstitution of phosphatidylcholine vesicles by dilution of phosphatidylcholine-sodium cholate mixed micelles. Biochemistry 1986, 25, 2597-2605.

(10) Gheriani-Gruszka, N.; Almog, S.; Biltonen, R. L.; Lichtenberg, D. Hydrolysis of phosphatidylcholine in phosphatidylcholine-cholate mixtures by porcine pancreatic phospholipase A2. J. Biol. Chem. 1988, 263, 11808-11813.

(11) Tamesue, N.; Inoue, T.; Juniper, K., Jr. Solubility of cholesterol in bile salt-lecithin model systems. Am. J. Dig. Dis. 1973, 18, 670-678.

(12) Hammad, M. A.; Müller, B. W. Increasing drug solubility by means of bile salt-phosphatidylcholine-based mixed micelles. Eur. J. Pharm. Biopharm. 1998, 46, 361-367.

(13) Chen, Y.; Lu, Y.; Chen, J.; Lai, J.; Sun, J.; Hu, F.; Wu, W. Enhanced bioavailability of the poorly water-soluble drug fenofibrate by using liposomes containing a bile salt. Int. J. Pharm. 2009, 376, 153-160.

(14) Walter, A.; Vinson, P. K.; Kaplun, A.; Talmon, Y. Intermediate structures in the cholate-phosphatidylcholine vesicle-micelle transition. Biophys. J. 1991, 60, 1315-1325.

(15) Madenci, D.; Salonen, A.; Schurtenberger, P.; Pedersen, J. S.; Egelhaaf, S. U. Simple model for the growth behaviour of mixed lecithin-bile salt micelles. Phys. Chem. Chem. Phys. 2011, 13, 31713178.

(16) Egelhaaf, S. U.; Schurtenberger, P. Shape transformations in the lecithin-bile salt system: from cylinders to vesicles. J. Phys. Chem. 1994, 98, 8560-8573.

(17) Meyuhas, D.; Bor, A.; Pinchuk, I.; Kaplun, A.; Talmon, Y.; Kozlov, M. M.; Lichtenberg, D. Effect of ionic strength on the selfassembly in mixtures of phosphatidylcholine and sodium cholate. $J$. Colloid Interface Sci. 1997, 188, 351-362.

(18) Mazer, N. A.; Benedek, G. B.; Carey, M. C. Quasielastic lightscattering studies of aqueous biliary lipid systems. Mixed micelle formation in bile salt-lecithin solutions. Biochemistry 1980, 19, 601615.

(19) Cohen, D. E.; Thurston, G. M.; Chamberlin, R. A.; Benedek, G. B.; Carey, M. C. Laser light scattering evidence for a common wormlike growth structure of mixed micelles in bile salt- and straightchain detergent-phosphatidylcholine aqueous systems: relevance to the micellar structure of bile. Biochemistry 1998, 37, 14798-14814.
(20) Hjelm, R. P.; Thiyagarajan, P.; Alkan-Onyuksel, H. Organization of phosphatidylcholine and bile salt in rodlike mixed micelles. J. Phys. Chem. 1992, 96, 8653-8661.

(21) Nichols, J. W.; Ozarowski, J. Sizing of lecithin-bile salt mixed micelles by size-exclusion high-performance liquid chromatography. Biochemistry 1990, 29, 4600-4606.

(22) Schurtenberger, P.; Scartazzini, R.; Luisi, P. L. Viscoelastic properties of polymer-like reverse micelles. Rheol. Acta 1989, 28, 372381.

(23) Shchipunov, Y. A. Lecithin organogel - a micellar system with unique properties. Colloids Surf., A 2001, 183, 541-554.

(24) Dreiss, C. A. Wormlike micelles: where do we stand? Recent developments, linear rheology and scattering techniques. Soft Matter 2007, 3, 956-970.

(25) Raghavan, S. R.; Kaler, E. W. Highly viscoelastic wormlike micellar solutions formed by cationic surfactants with long unsaturated tails. Langmuir 2000, 17, 300-306.

(26) Tung, S.-H.; Huang, Y.-E.; Raghavan, S. R. A new reverse wormlike micellar system: mixtures of bile salt and lecithin in organic liquids. J. Am. Chem. Soc. 2006, 128, 5751-5756.

(27) Njauw, C.-W.; Cheng, C.-Y.; Ivanov, V. A.; Khokhlov, A. R.; Tung, S.-H. Molecular interactions between lecithin and bile salts/ acids in oils and their effects on reverse micellization. Langmuir 2013, $29,3879-3888$.

(28) Lo Nostro, P.; Ninham, B. W. Hofmeister phenomena: an update on ion specificity in biology. Chem. Rev. 2012, 112, 22862322.

(29) Lee, T.; Chen, J. G. Biomimetic gallstone formation: crystallization of calcium carbonate by the evolving taurocholatelecithin-cholesterol complex lipid system. Cryst. Growth Des. 2009, 9, 3737-3748.

(30) Admirand, W. H.; Small, D. M. The physicochemical basis of cholesterol gallstone formation in man. J. Clin. Invest. 1968, 47, 104352.

(31) Raut, S.; Bhadoriya, S. S.; Uplanchiwar, V.; Mishra, V.; Gahane, A.; Jain, S. K. Lecithin organogel: a unique micellar system for the delivery of bioactive agents in the treatment of skin aging. Acta Pharm. Sin. B 2012, 2, 8-15.

(32) Elnaggar, Y. S. R.; El-Refaie, W. M.; El-Massik, M. A.; Abdallah, O. Y. Lecithin-based nanostructured gels for skin delivery: an update on state of art and recent applications. J. Controlled Release 2014, 180, $10-24$.

(33) Jeng, U.-S.; Su, C. H.; Su, C.-J.; Liao, K.-F.; Chuang, W.-T.; Lai, Y.-H.; Chang, J.-W.; Chen, Y.-J.; Huang, Y.-S.; Lee, M.-T.; Yu, K.-L.; Lin, J.-M.; Liu, D.-G.; Chang, C.-F.; Liu, C.-Y.; Chang, C.-H.; Liang, K. $\mathrm{S}$. A small/wide-angle X-ray scattering instrument for structural characterization of air-liquid interfaces, thin films and bulk specimens. J. Appl. Crystallogr. 2010, 43, 110-121.

(34) Kline, S. Reduction and analysis of SANS and USANS data using IGOR Pro. J. Appl. Crystallogr. 2006, 39, 895-900.

(35) Pedersen, J. S. Analysis of small-angle scattering data from colloids and polymer solutions: modeling and least-squares fitting. $A d v$. Colloid Interface Sci. 1997, 70, 171-210.

(36) Pedersen, J. S.; Schurtenberger, P. Scattering functions of semiflexible polymers with and without excluded volume effects. Macromolecules 1996, 29, 7602-7612.

(37) Chen, W.-R; Butler, P. D.; Magid, L. J. Incorporating intermicellar interactions in the fitting of SANS data from cationic wormlike micelles. Langmuir 2006, 22, 6539-6548.

(38) Livsey, I. Neutron scattering from concentric cylinders. Intraparticle interference function and radius of gyration. J. Chem. Soc., Faraday Trans. 2 1987, 83, 1445-1452.

(39) Small, D. M.; Admirand, W. Solubility of bile salts. Nature 1969, 221, 265-267.

(40) Garidel, P.; Hildebrand, A.; Neubert, R.; Blume, A. Thermodynamic characterization of bile salt aggregation as a function of temperature and ionic strength using isothermal titration calorimetry. Langmuir 2000, 16, 5267-5275. 
(41) Evans, D. F.; Wennerstrom, H. The Colloidal Domain: Where Physics, Chemistry, Biology, and Technology Meet, 2nd ed.; Wiley-VCH: New York, 1999.

(42) Kunz, W.; Henle, J.; Ninham, B. W. Zur Lehre von der Wirkung der Salze (about the science of the effect of salts): Franz Hofmeister's historical papers. Curr. Opin. Colloid Interface Sci. 2004, 9, 19-37.

(43) Hofmann, A. F.; Mysels, K. J. Bile acid solubility and precipitation in vitro and in vivo: the role of conjugation, $\mathrm{pH}$, and $\mathrm{Ca}^{2+}$ ions. J. Lipid Res. 1992, 33, 617-26.

(44) Gunnarsson, G.; Joensson, B.; Wennerstroem, H. Surfactant association into micelles. An electrostatic approach. J. Phys. Chem. 1980, 84, 3114-3121.

(45) Akutsu, H.; Seelig, J. Interaction of metal ions with phosphatidylcholine bilayer membranes. Biochemistry 1981, 20, 7366-7373.

(46) Lee, H.-Y.; Diehn, K. K.; Ko, S. W.; Tung, S.-H.; Raghavan, S. R. Can simple salts influence self-assembly in oil? Multivalent cations as efficient gelators of lecithin organosols. Langmuir 2010, 26, 1383113838.

(47) Schurtenberger, P.; Mazer, N.; Kaenzig, W. Micelle to vesicle transition in aqueous solutions of bile salt and lecithin. J. Phys. Chem. 1985, 89, 1042-1049.

(48) Shrestha, L. K.; Yamamoto, M.; Arima, S.; Aramaki, K. Chargefree reverse wormlike micelles in nonaqueous media. Langmuir 2011, 27, 2340-2348.

(49) Glatter, O. The interpretation of real-space information from small-angle scattering experiments. J. Appl. Crystallogr. 1979, 12, 166175.

(50) Brunner-Popela, J.; Glatter, O. Small-angle scattering of interacting particles. I. Basic principles of a global evaluation technique. J. Appl. Crystallogr. 1997, 30, 431-442.

(51) Varade, D.; Ushiyama, K.; Shrestha, L. K.; Aramaki, K. Wormlike micelles in Tween-80/ $\mathrm{C}_{\mathrm{m}} \mathrm{EO}_{3}$ mixed nonionic surfactant systems in aqueous media. J. Colloid Interface Sci. 2007, 312, 489-497. (52) May, A.; Pasc, A.; Stébé, M. J.; Gutiérrez, J. M.; Porras, M.; Blin, J. L. Tailored jeffamine molecular tools for ordering mesoporous silica. Langmuir 2012, 28, 9816-9824.

(53) Shrestha, L. K.; Shrestha, R. G.; Aramaki, K.; Yoshikawa, G.; Ariga, K. Demonstration of solvent-induced one-dimensional nonionic reverse micelle growth. J. Phys. Chem. Lett. 2013, 4, 2585-2590.

(54) Israelachvili, J. N. Intermolecular and Surface Forces, 3rd ed.; Academic Press: San Diego, 2011.

(55) Zangi, R.; Berne, B. J. Aggregation and dispersion of small hydrophobic particles in aqueous electrolyte solutions. J. Phys. Chem. B 2006, 110, 22736-22741.

(56) Carey, M. C.; Small, D. M. Micellar properties of dihydroxy and trihydroxy bile salts: effects of counterion and temperature. J. Colloid Interface Sci. 1969, 31, 382-396. 